\title{
Bunch Profiles in RHIC from CNI Polarimeter Target Scans
}

High Energy Physics Division Argonne National Laboratory 


\section{About Argonne National Laboratory}

Argonne is operated by The University of Chicago for the U.S. Department of Energy Office of Science, under contract W-31-109-Eng-38. The Laboratory's main facility is outside Chicago, at 9700 South Cass Avenue, Argonne, Illinois 60439. For information about Argonne and its pioneering science and technology programs, see www.anl.gov.

\section{Availability of This Report}

This report is available, at no cost, at http://www.osti.gov/bridge. It is also available on paper to U.S. Department of Energy and its contractors, for a processing fee, from:

U.S. Department of Energy

Office of Scientific and Technical Information

P.O. Box 62

Oak Ridge, TN 37831-0062

phone (865) 576-8401

fax (865) 576-5728

reports@adonis.osti.gov

\footnotetext{
Disclaimer

This report was prepared as an account of work sponsored by an agency of the United States Government. Neither the United States Government nor any agency thereof, nor The University of Chicago, nor any of their employees or officers, makes any warranty, express or implied, or assumes any legal liability or responsibility for the accuracy, completeness, or usefulness of any information, apparatus, product, or process disclosed, or represents that its use would not infringe privately owned rights. Reference herein to any specific commercial product, process, or service by trade name, trademark, manufacturer, or otherwise, does not necessarily constitute or imply its endorsement, recommendation, or favoring by the United States Government or any agency thereof. The views and opinions of document authors expressed herein do not necessarily state or reflect those of the United States Government or any agency thereof, Argonne National Laboratory, or The University of Chicago.
} 
ANL-HEP-TR-05-033

$\mathrm{C}-\mathrm{A} / \mathrm{AP} / 206$

\title{
Bunch Profiles in RHIC from CNI
}

\section{Polarimeter Target Scans}

\author{
H. Spinka \\ Argonne National Laboratory \\ 13 May 2005
}

During the RHIC run-4 polarized proton operation, the intensity and polarization profiles were measured by scanning the Coulomb-nuclear interference $(\mathrm{CNI})$ polarimeter targets across the beam. The scans reported here were all performed on flattop at beam momenta $\sim 100 \mathrm{GeV} / \mathrm{c}$, and all but one was a vertical scan, since several of the vertical polarimeter targets had broken by the time of the measurements. This note summarizes some analyses of these profiles. Wall current monitor data are compared to these results for one beam and fill.

\section{Transverse Intensity Profile Measurements}

The raw data from the $\mathrm{p}+\mathrm{C}$ CNI polarimeters (Ref. 1) included the summed counts passing cuts from each of the six silicon-strip detectors in the relevant polarimeter (left, right, and $\pm 45^{\circ}$ and $\pm 135^{\circ}$ to the vertical) for each bunch as a function of target position. The polarimeters were operated in the scaler mode for these results, and the time of the measurements was also recorded. There were five scans performed at flattop in run-4 - one each in the vertical direction for the yellow and blue beams for fills 5236 and 5341. The fifth scan was in the horizontal direction for the blue beam in fill 5236.

For intensity profiles, the sum of counts from the six detectors was divided by the elapsed time. The centroid and rms width of the profiles were computed for each bunch. Histograms of these quantities for the yellow beam vertical scan in fill 5236 are shown in Fig. 1. The two scans in the blue beam for fill 5236 are shown in Fig. 2, and the two vertical scans in fill 5341 are given in Fig. 3. From these plots, it is clear that there is at least one bunch with considerably different properties in each scan. There are several such bunches in the blue beam for both fills 5236 and 5341, while there seems to be two classes of bunch widths in the yellow beam in fill 5341.

In each of the five scans, bunch \# 21 (among 1 - 120, with the last ones being the abort gap, and only the odd bunches "populated") has an anomalously large width. This bunch corresponds to the one that is perturbed in order to measure the tune of the beam during acceleration in RHIC. Its profile is usually much different from the other bunches; see Fig. 4. 

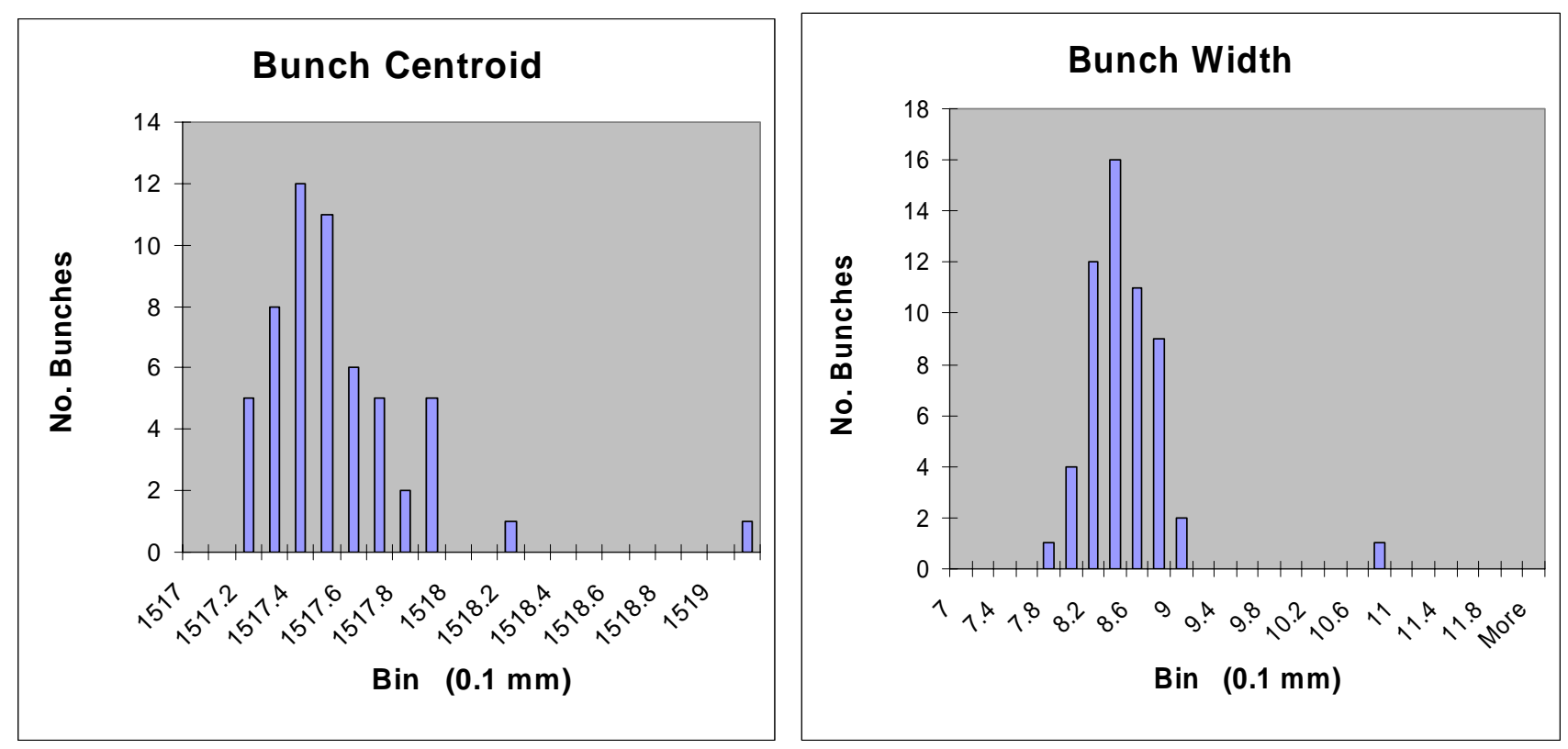

Fig. 1. Histograms of bunch centroids and rms widths from the vertical scan in the yellow beam in fill 5236. This centroid distribution is believed to be artificially wide due to an incomplete scan over the full height of the beam (see Fig. 4).

The horizontal scales for the centroids for all five scans in Figs. 1-3 are the same, corresponding to a $0.2 \mathrm{~mm}$ range. These distributions are all quite narrow except for the yellow beam vertical scan in fill 5236 (Fig. 1). However, in this case the scan did not completely cover the beam profile, as seen in Fig. 4. It is expected that the centroid distribution in this case would have been considerably narrower if there had been full coverage. Thus, all bunches in a particular fill and beam appear to have identical centroids to better than $0.1 \mathrm{~mm}$ for the scans performed and studied in this note.

On the other hand, the bunch width distributions generally show differences from fill to fill and beam to beam. Excluding bunch \# 21, the yellow beam vertical scan in fill 5236 appears to show a relatively narrow distribution of rms widths. This distribution may have been narrower had a scan with complete coverage of the beam profile been performed. Ideally, all the scans should have looked like this one (or narrower). Figure 4 shows that the bunch shapes (except \# 21) appear to be similar, with some differences in beam intensity, though some bunches are a bit wider than others.

In contrast, the blue beam scans for both fills have a few bunches that are considerably wider than average. The widest and narrowest bunches for the blue beam for fill 5236 are shown in Fig. 5, and for fill 5341 are given in Fig. 6. The widest bunches can be seen to have a different shape from the rest, including some that seem to have a dip in intensity in the center! Furthermore, most have somewhat less total beam than the narrower bunches. It should be noted that some "structure" in these profiles is probably caused by the coarse time bins of $1 \mathrm{sec}$ and measurement times as short as $12 \mathrm{sec}$. Thus, "round-off" errors are not negligible, especially in the higher intensity portions of the scans, which had the shortest measurement times. 

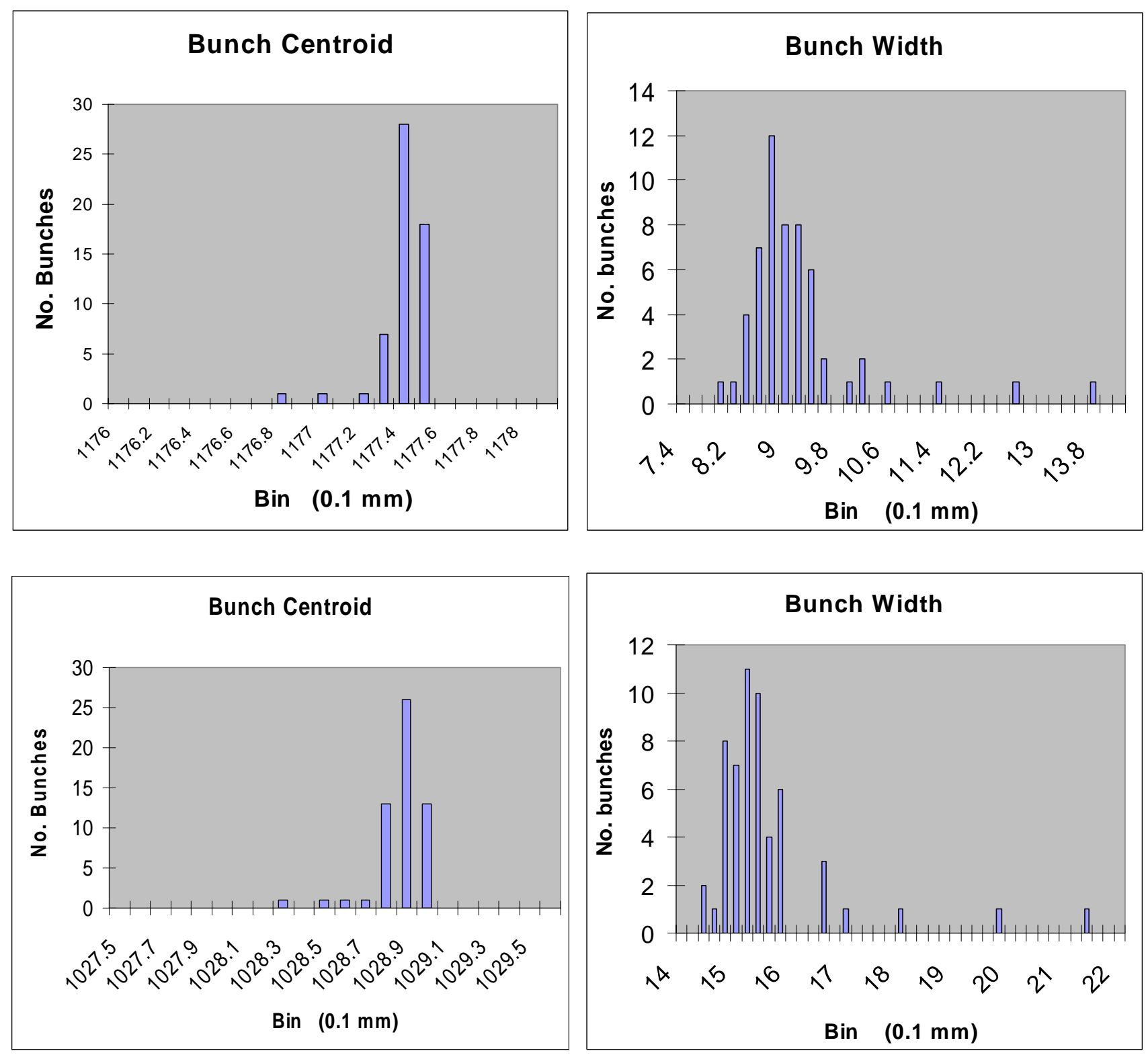

Fig. 2. Histograms of bunch centroids and rms widths from the horizontal (upper) and vertical (lower) scans in the blue beam in fill 5236.

The yellow beam vertical scan for fill 5341 in Fig. 3 seems to show a bi-modal width distribution (plus bunch \# 21, which is much wider). Excluding bunch \#21, the four widest bunches all appear to have essentially the same profile and width, while the five narrowest bunches seem to have the same shape with some differences in total intensity. It should be recalled that data for all bunches at a particular target setting were collected simultaneously. Also, in this case the total intensity in the various bunches is nearly the same, with the wider bunches containing perhaps $15-20 \%$ less beam. None of the other scans showed such behavior. 

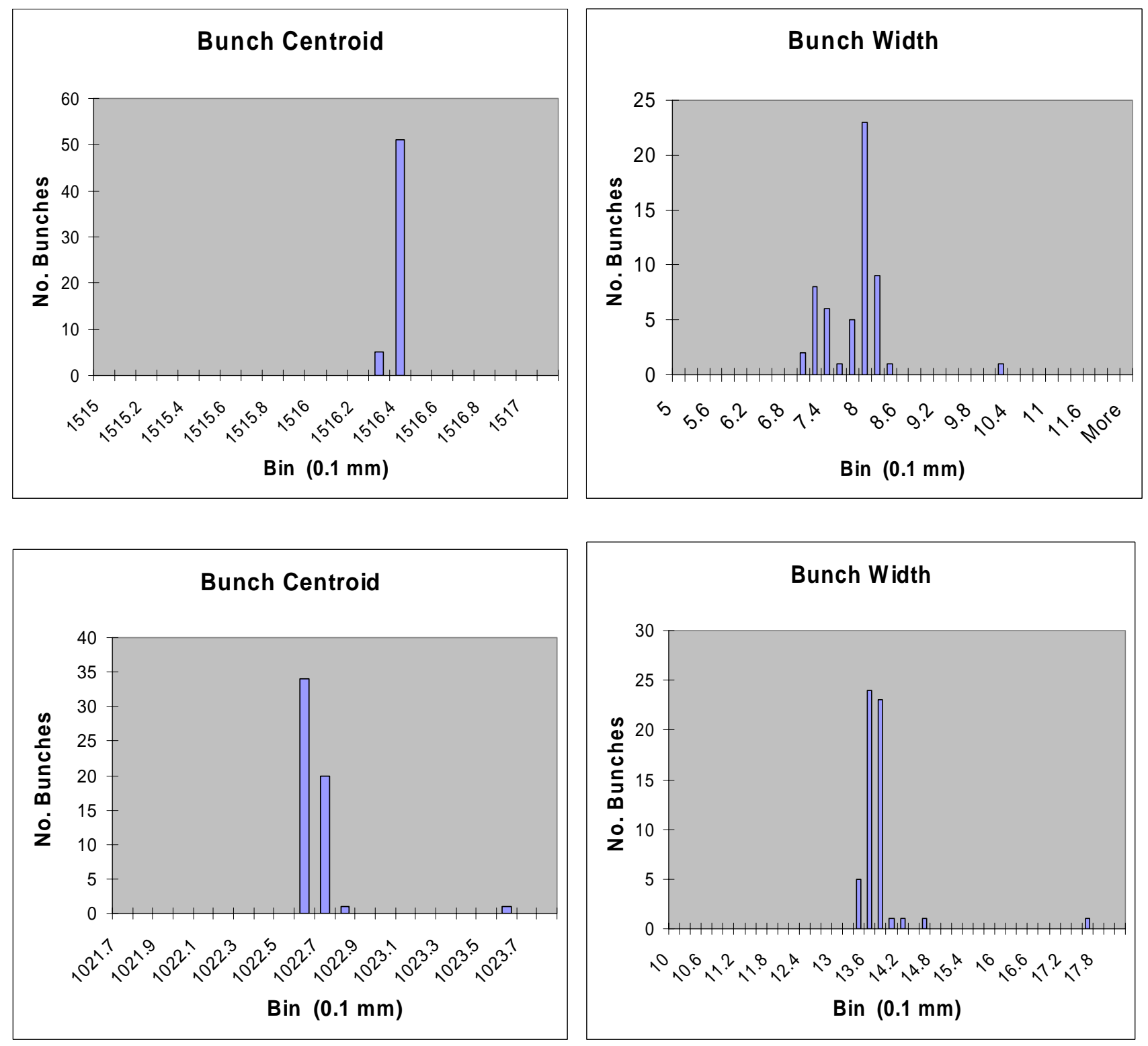

Fig. 3. Histograms of bunch centroids and rms widths from the yellow (upper) and blue (lower) vertical scans in fill 5341. Note the bi-modal distribution for the yellow beam widths.

For the blue beam in fill 5236, both horizontal and vertical beam scan data are available. Figure 7 shows that a strong correlation exists between the rms widths calculated for the individual bunches. However, the widths are not proportional, or equivalently, the correlation does not pass through the origin. Uncertainties on the widths have not been calculated for a number of reasons. For example, the round-off problem in the measurement time would make the calculation very complicated. Also, there was beam loss during each polarimeter measurement for each target position. Finally, the accuracy of the target location compared to the digital readout was unknown. The variation of the individual points from a line through them, especially at small widths, probably provides the best estimate of the uncertainties. 


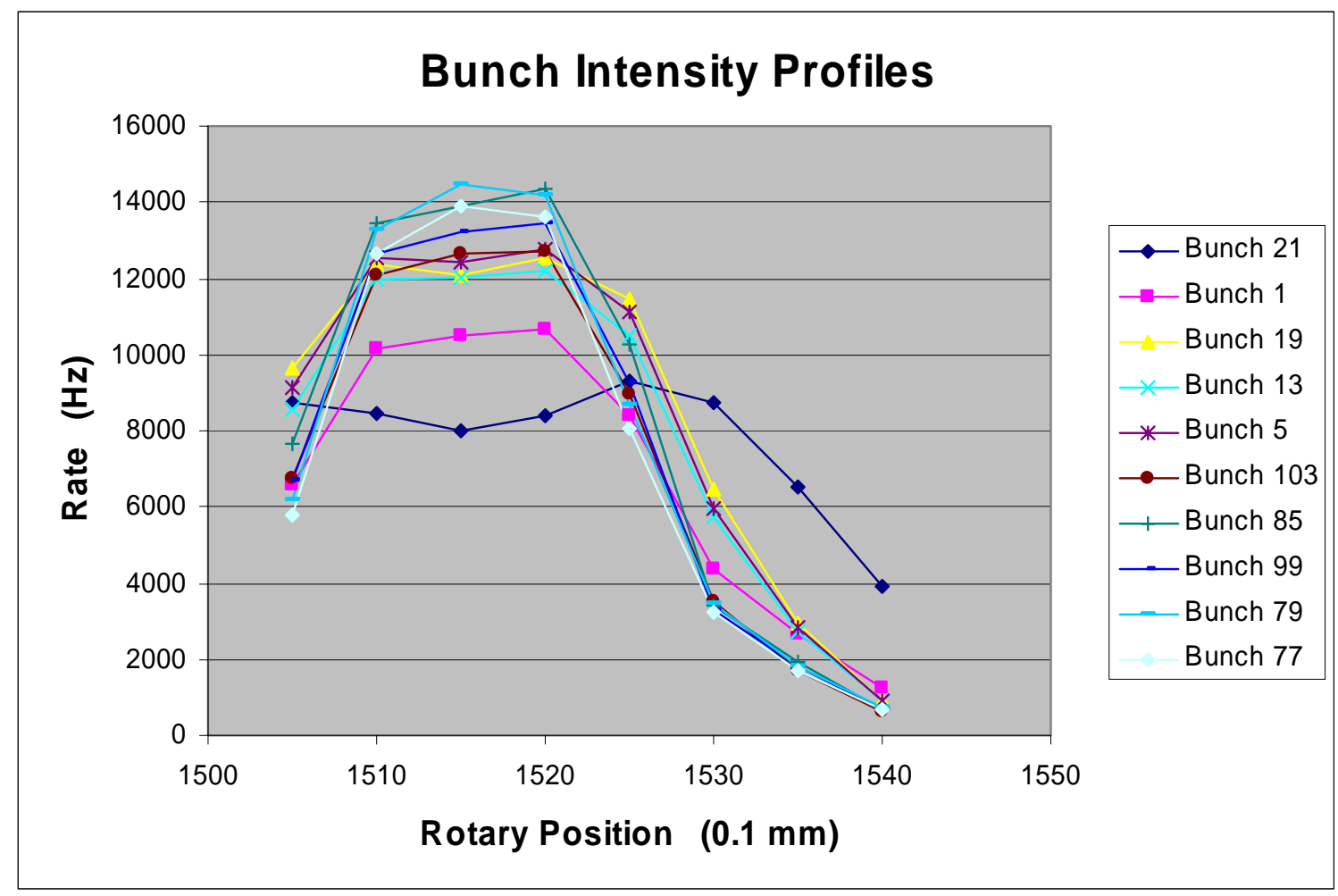

Fig. 4. Intensity profiles for the five widest and five narrowest bunches (widest to narrowest in the plot legend) for the vertical scan in the yellow beam in fill 5236. The shape of all bunches (except 21) appears to be roughly the same.

For reference, the rms widths $\left(\sigma_{\mathrm{w}}\right)$ of the bunch profiles have been tabulated below. These show considerable variation, and the results suggest there is a fill to fill and beam to beam difference in bunch shape distributions:

Fill 5236, yellow beam, vscan, $\sigma_{\mathrm{w}}=0.27 \mathrm{~mm}, \sigma_{\mathrm{w}} / \mathrm{av}=0.032$

Fill 5236, blue beam, hscan, $\sigma_{\mathrm{w}}=0.36 \mathrm{~mm}, \sigma_{\mathrm{w}} / \mathrm{av}=0.040$

Fill 5236, blue beam, vscan, $\sigma_{\mathrm{w}}=0.36 \mathrm{~mm}, \sigma_{\mathrm{w}} / \mathrm{av}=0.024$

Fill 5341, yellow beam, vscan, $\sigma_{\mathrm{w}}=0.38 \mathrm{~mm}, \sigma_{\mathrm{w}} / \mathrm{av}=0.049$

Fill 5341, blue beam, vscan, $\sigma_{\mathrm{w}}=0.14 \mathrm{~mm}, \sigma_{\mathrm{w}} / \mathrm{av}=0.010$

The number of wide bunches omitted from the estimation of $\sigma_{\mathrm{w}}$ was $1,7,7,1$, and 3 , respectively, including bunch \# 21 in all cases.

\section{Longitudinal Intensity Profile Studies}

Wall current monitor (WCM) measurements (Ref. 2) of the longitudinal bunch profiles for the blue beam in fill 5236 were analyzed and the results correlated with those from the transverse profiles.

Five sets of WCM data were used, all from the time of the horizontal CNI scan in the blue beam. The 

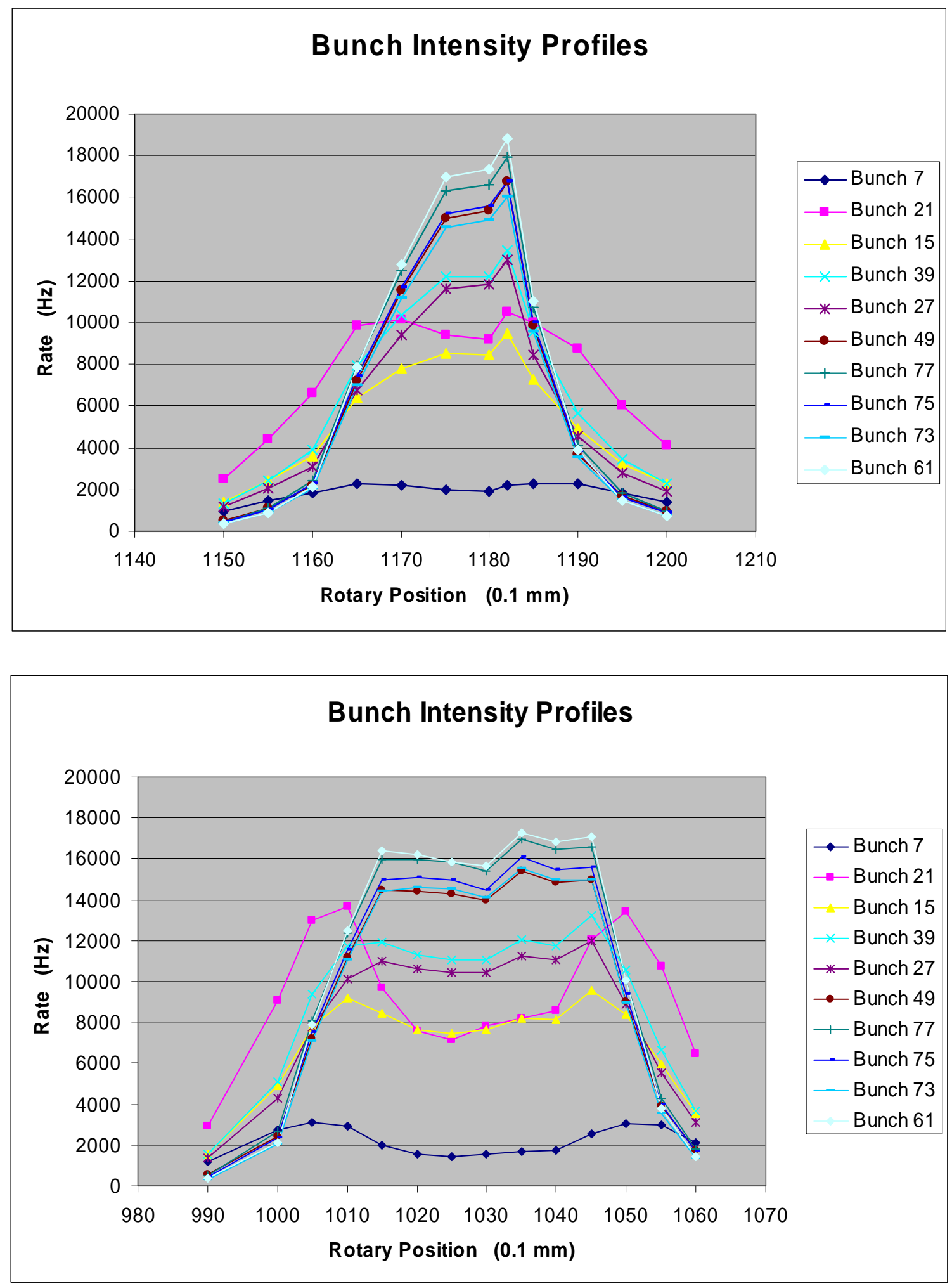

Fig. 5. Intensity profiles for the five widest and five narrowest bunches (widest to narrowest in the plot legend) for the horizontal (upper) and vertical (lower) scans in the blue beam in fill 5236. The same bunches are plotted for both scans for comparison. 

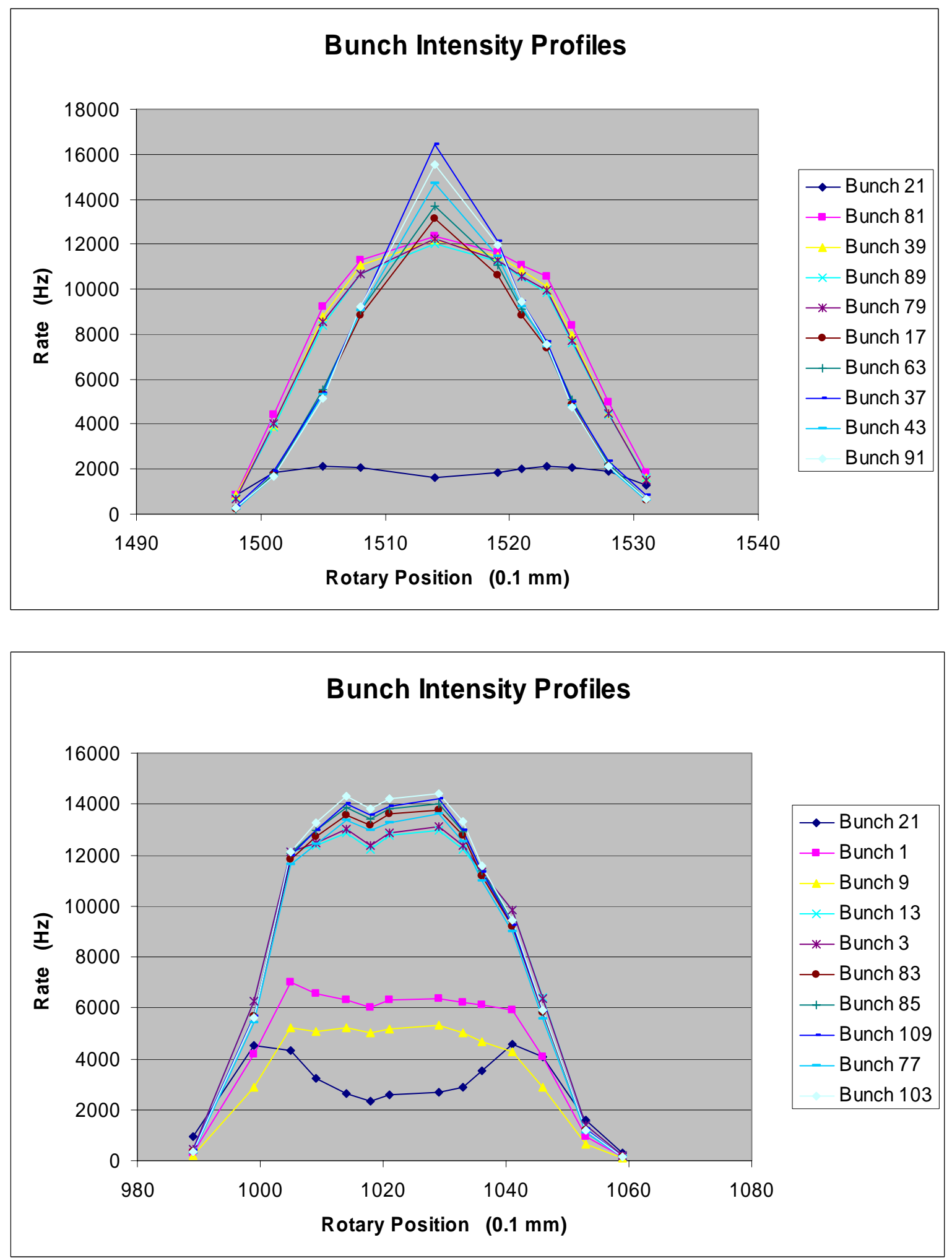

Fig. 6. Intensity profiles for the five widest and five narrowest bunches (widest to narrowest in the plot legend) for the yellow (upper) and blue (lower) vertical scans in fill 5341. Note that different bunches are plotted for the two scans. 


\section{Fill 5236 - Blue Beam}

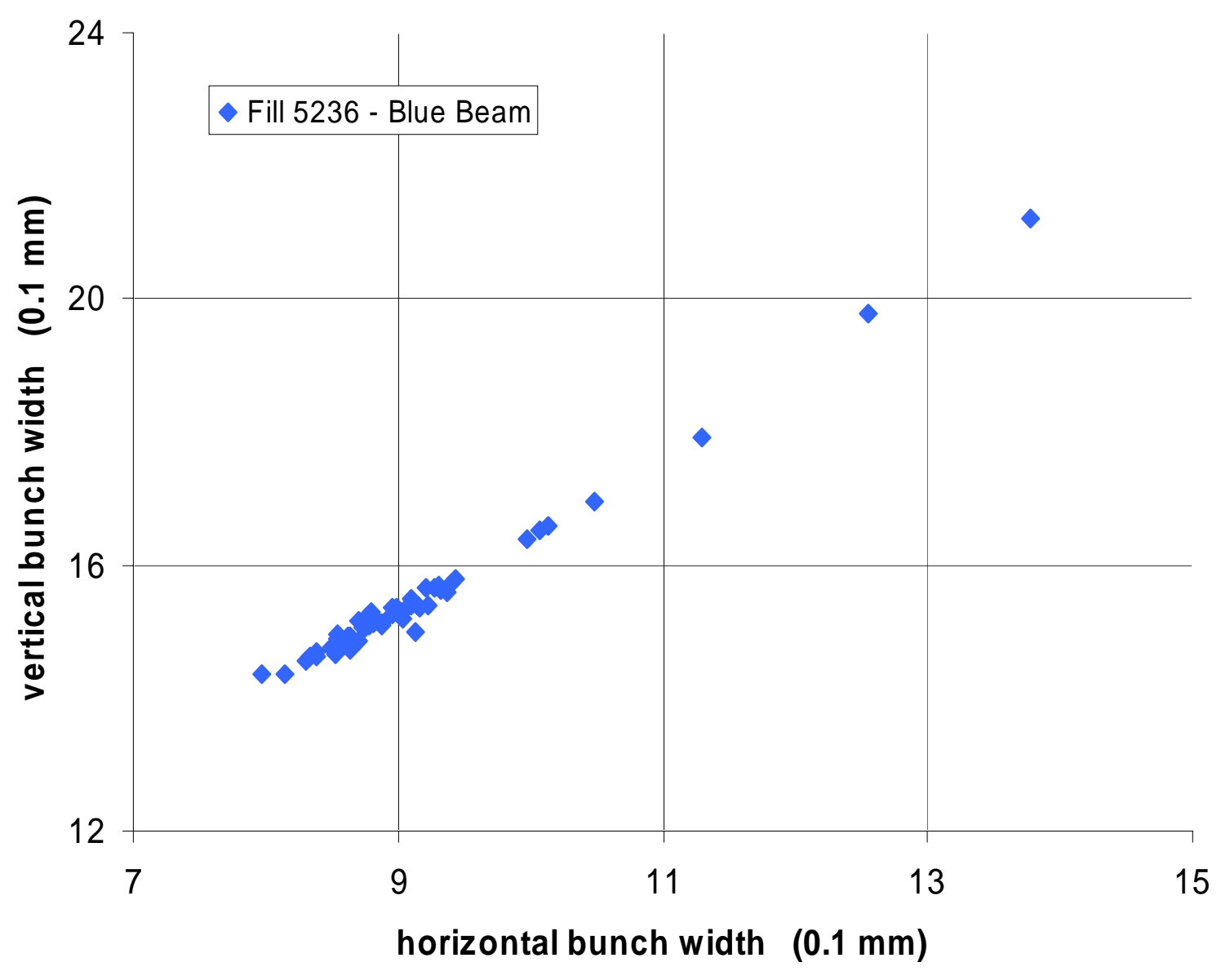

Fig. 7. Correlation of vertical and horizontal bunch widths measured with the blue beam in fill 5236. A strong correlation is observed.

five sets appeared consistent, so averages were computed and these were used in various studies described below.

Four longitudinal profiles from a single data set are shown in Fig. 8. Two bunches $(\# 9,111)$ seem to have an asymmetric distribution, while bunch \# 21 has a "narrow," symmetric shape. The fourth example (\# 11) is considerably wider. The low intensity bunch \# 7 is shown in Fig. 9; discrete ADC values are apparent with a magnitude $\sim 0.022$. Also shown in Fig. 9 is the widest bunch (\# 105). Of the 56 bunches in the blue beam for fill 5236, 24 had approximately symmetric and narrow shapes, 20 had offset maxima similar to bunches \# 9 and 111, and 12 bunches were wide. There was no discernable pattern to the distribution of these different types of bunches within the fill. 

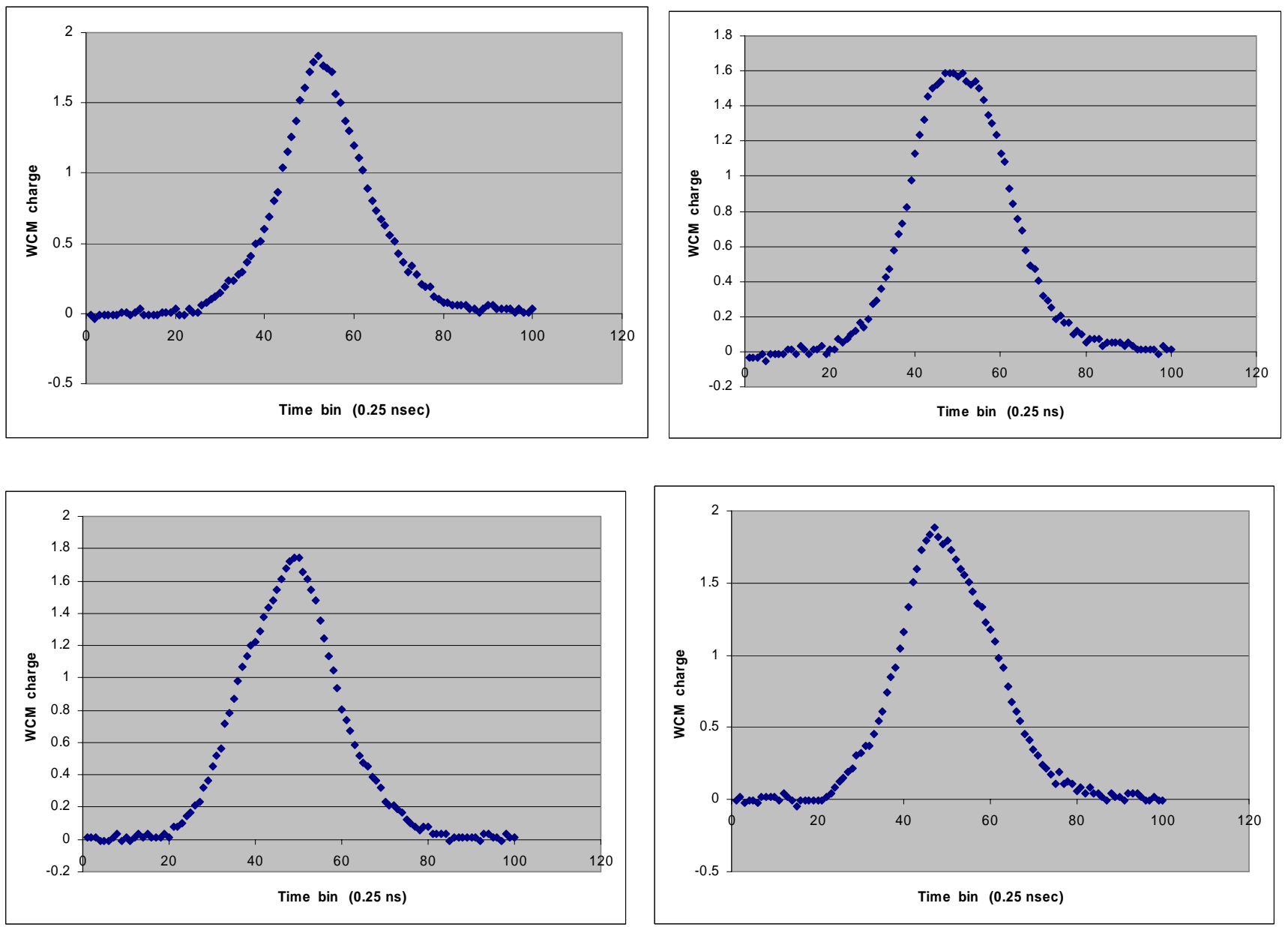

Fig. 8. Longitudinal intensity profiles after pedestal subtraction for bunches \# 21 (upper left), \# 11 (upper right), \# 9 (lower left), and \# 111 (lower right). The same WCM data set was used for all of these plots. In the lower figures, the peak seems offset from the center about 2 time bins or $15 \mathrm{~cm}$.
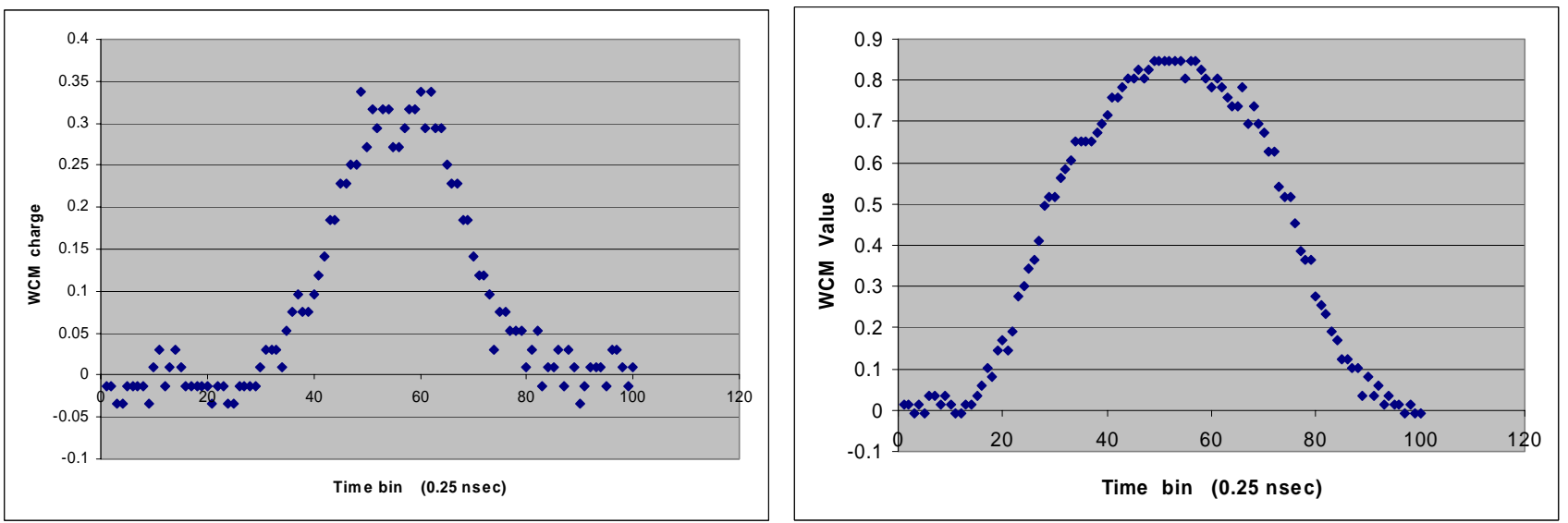

Fig. 9. Longitudinal intensity profiles for the low intensity bunch (\# 7) and the widest bunch (\# 105). 
The "area" of each bunch was computed by adding WCM data for 100 time bins of 0.25 nsec width, roughly centered on the bunch. The pedestal was estimated as the mean of the sums for the preceding 100 time bins and for the100 following time bins, and this was subtracted from the area. The mean time and rms width were also computed from the pedestal-subtracted data.

The area from the WCM data is expected to be proportional to the number of particles in the bunch. The area from the CNI scan results should also be roughly proportional to the number of particles. Beam losses during the scans and imprecise times for data collection may have caused deviations from true proportionality, however. A correlation of the two areas is shown in Fig. 10. A reasonably good correlation is observed, with a few "outliers." The five narrowest bunches from the CNI measurements (see Fig. 5) are given with yellow triangles, and all lie systematically above the average. Likewise, the widest bunches shown as red diamonds all lie below the average and several deviate significantly, including bunches \# 7 and 21.

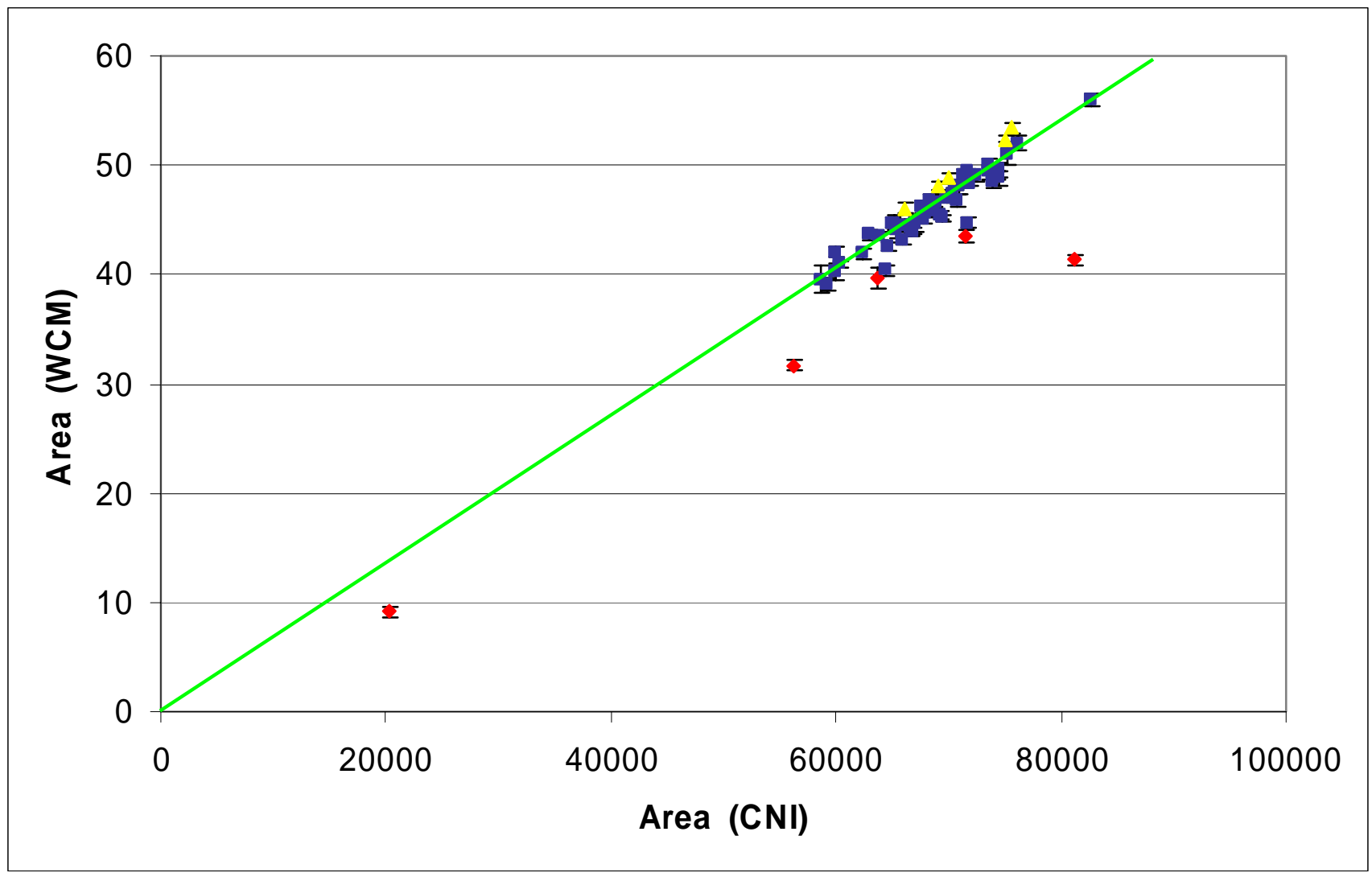

Fig. 10. Correlation of the WCM and CNI bunch areas. The yellow triangles correspond to the narrowest transverse widths as measured in the CNI scans, and the red diamonds correspond to the widest widths; see Fig. 5. The green line is placed to guide the eye.

Two tests were performed to see if the correlation could be improved. One subtracted $5 \mathrm{sec}$ from each CNI measurement time - see Fig. 11. The other assumed a loss of CNI counts proportional to the rate, so there would be a $25 \%$ loss at the highest rates; see Fig. 12. Neither of these major changes solved the deviation from proportionality, and the high intensity CNI bunches appear overly corrected 


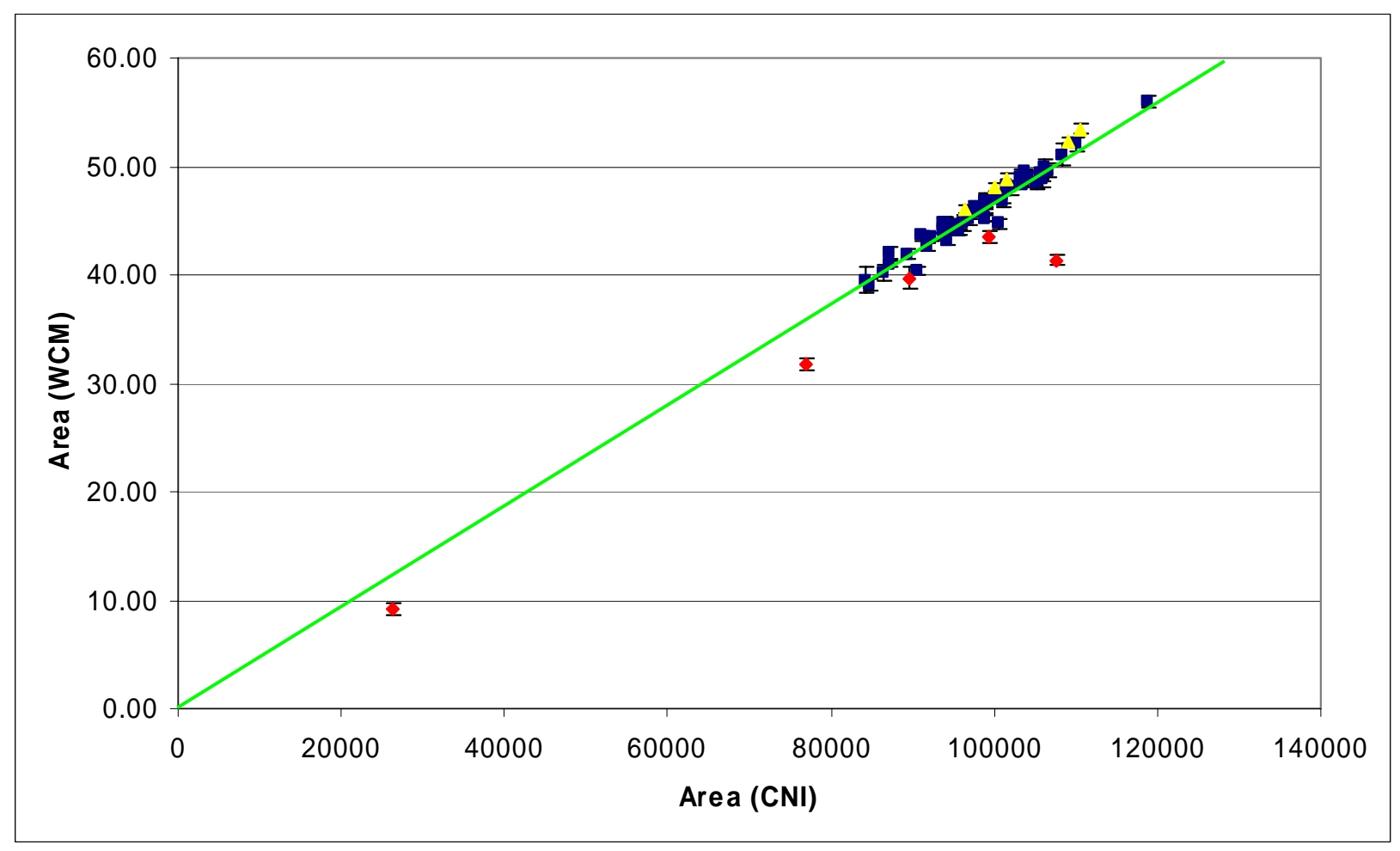

Fig. 11. . Correlation of the WCM and CNI bunch areas with CNI measurement times reduced by 5 sec. The yellow triangles correspond to the narrowest transverse widths as measured in the CNI scans, and the red diamonds correspond to the widest widths; see Fig. 5. The green line is placed to guide the eye.

in Fig. 12. Thus, the conclusion is that some unknown instrumental effect causes a width-dependence to the measured area in either the WCM or the CNI polarimeter (or both).

A least squares fit to the mean bunch positions was made assuming a constant bunch spacing. The uncertainties on the position were found from the variation among the five data sets, and a good fit was obtained $\left(\chi^{2} /\right.$ d.f. $=0.875$ for 56 positions). The distribution of deviations from the fit is shown in Fig. 13, excluding bunch \# 7 which exhibited a large variation because of its low amplitude. Thus, it is concluded that the mean longitudinal bunch centroids are very close to a constant spacing.

Finally, the rms widths of the longitudinal and transverse bunch profiles are plotted in Fig. 14. There is some very weak evidence for a correlation, with larger longitudinal widths corresponding to narrower transverse widths. Note bunch \# 105 deviates considerably from the others; see also Fig. 9. 


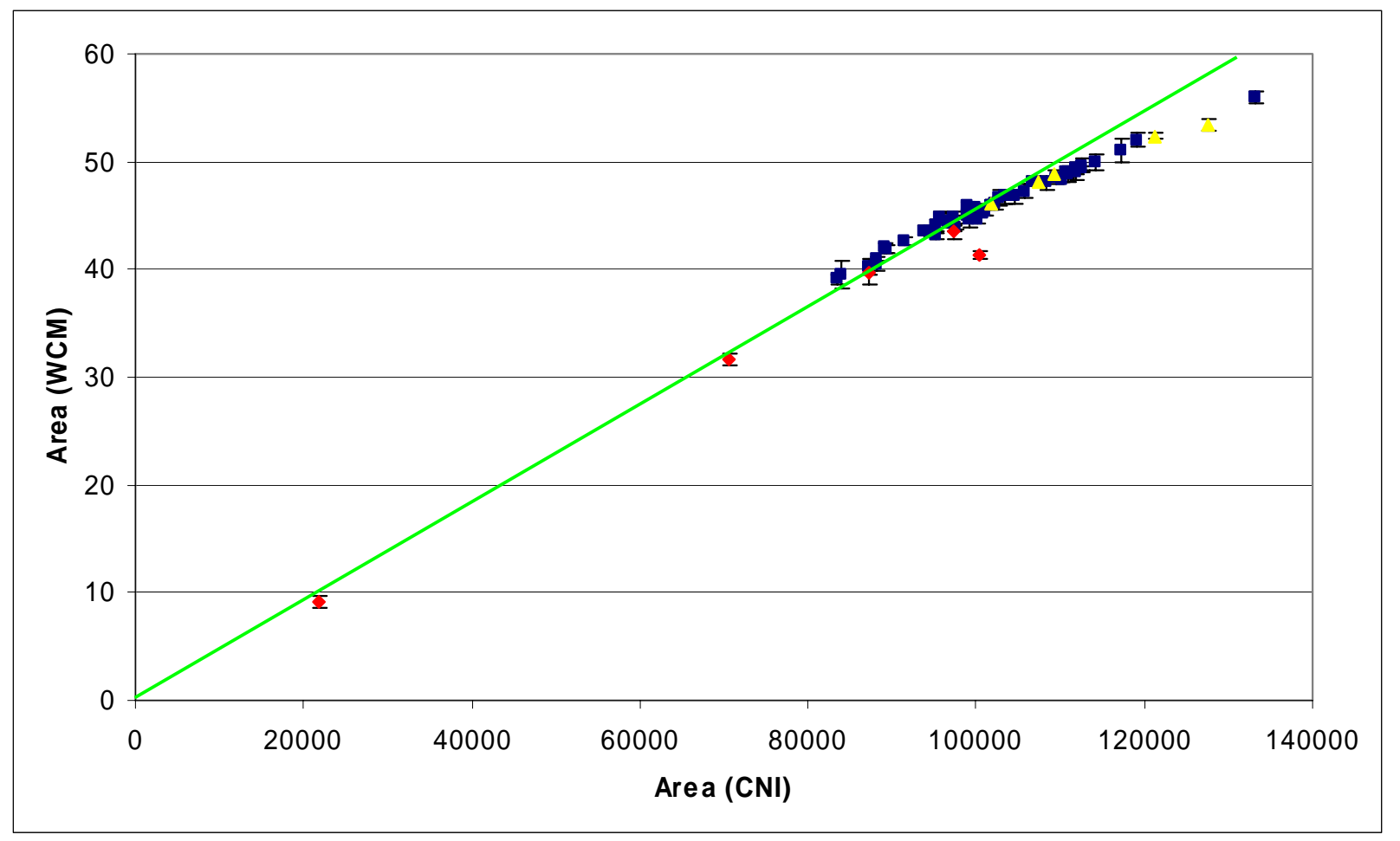

Fig. 12. Correlation of the WCM and CNI bunch areas, assuming a loss of CNI counts proportional to the rate ( $25 \%$ loss at $20 \mathrm{kHz})$. The yellow triangles correspond to the narrowest transverse widths as measured in the CNI scans, and the red diamonds correspond to the widest widths; see Fig. 5. The green line is placed to guide the eye. Note the distortion at large areas.

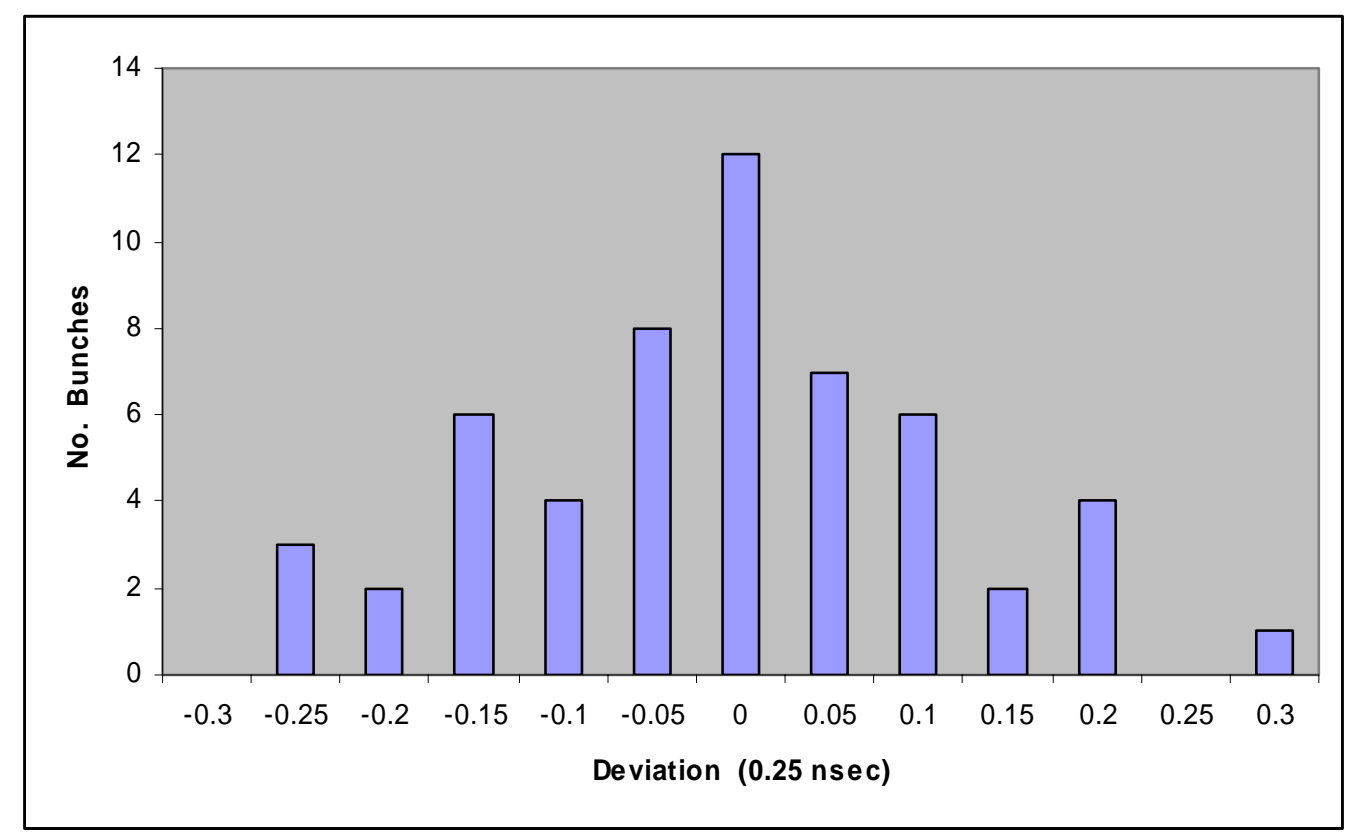

Fig. 13. Histogram of the deviations of the longitudinal bunch centroids from the least squares fit line. 


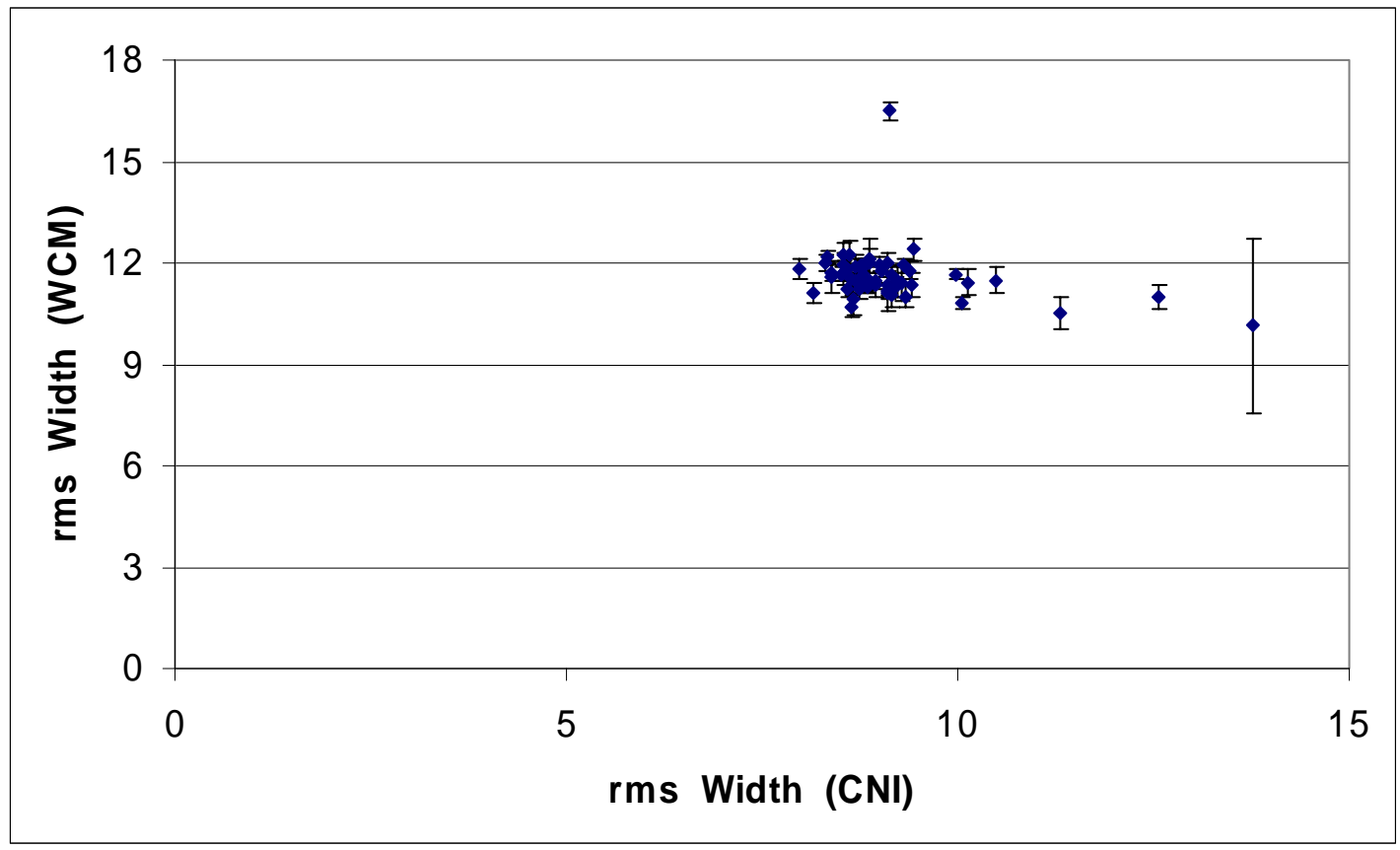

Fig. 14. Correlation of rms widths for WCM and CNI bunch measurements.

\section{Polarization Profile Measurements}

The blue beam horizontal scan for fill 5236 was analyzed to search for differences in polarization correlated with bunch width. This scan was taken near the beginning of the fill, on flattop, in two passes from small to large position values. The 56 bunches were separated into four sets of 14 , each with $7+$ and $7-$ bunches, approximately according to width. The asymmetry results calculated with the standard square root formula are shown in Fig. 15. Statistical uncertainties on each point are about $\pm 0.0008-0.0016$, and half that for the average. The errors are largest at the edges of the bunches. The average asymmetries seem somewhat larger for narrow bunches than for wider ones, and each of the four sets is consistent with being constant as a function of position:

$$
\begin{array}{rlrl}
<\varepsilon_{\text {narrow }}>=(5.39 \pm 0.24) \times 10^{-3} & \chi^{2}=11.4 \\
<\varepsilon_{\text {mid_narr }}>=(5.73 \pm 0.25) \times 10^{-3} & =9.9 \\
<\varepsilon_{\text {mid_wide }}>=(5.03 \pm 0.24) \times 10^{-3} & =18.2 \\
<\varepsilon_{\text {wide }}>=(4.92 \pm 0.24) \times 10^{-3} & & =10.7
\end{array}
$$

for 11 degrees of freedom. The weighted average is $(5.26 \pm 0.12) \times 10^{-3}$ with $\chi^{2} /$ d.f. $=2.24$. The bunch numbers used for the four sets are: narrow (pos $=33,49,61,73,77,85,101$; neg $=35,55,71$, $75,83,103,107$ ), mid-narrow ( $\operatorname{pos}=1,41,53,69,81,97,109$; neg $=47,59,63,67,79,87,91$ ), midwide (pos $=9,29,37,57,65,89,93$; neg $=11,23,43,51,95,99,111)$, and wide (pos $=5,13,17,21$, $25,45,105 ;$ neg $=3,7,15,19,27,31,39$ ). In addition, the summed counts from all positions except 


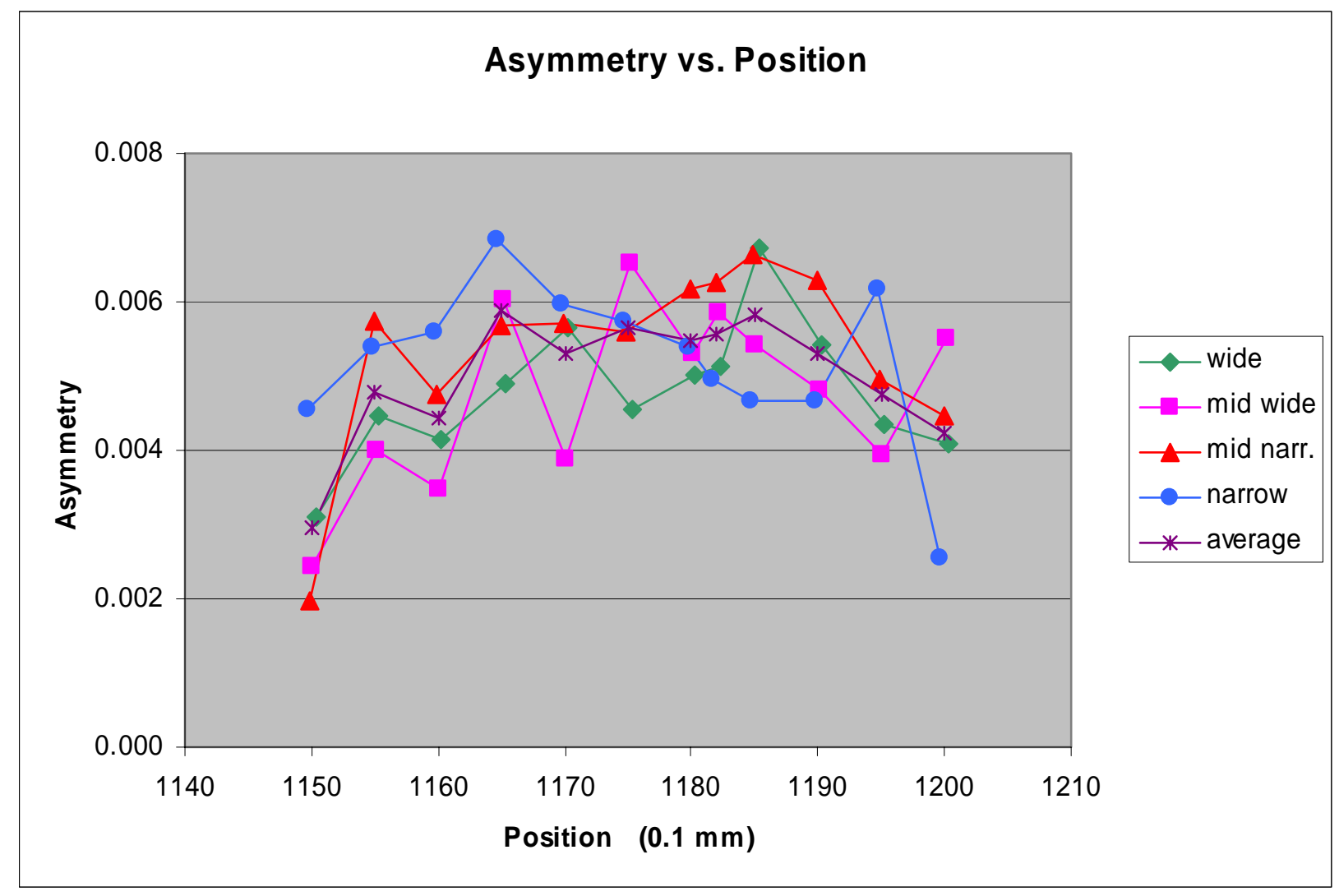

Fig. 15. Left-right asymmetries, calculated with the square root formula, are shown for four sets of bunches, selected approximately according to width. The individual statistical uncertainties are typically $\pm 0.0008-0.0016$.

the ends $(1150,1200)$ were used to calculate asymmetries; pairs of bunches were used. The widest bunches for + and - polarization were one pair, the next widest were another pair, etc. The results are shown in Fig. 16, and there is also some indication of asymmetry dependence with bunch width.

A vertical scan in the same beam was taken somewhat later in fill 5236. With the same set of bunches:

$$
\begin{array}{lrl}
<\varepsilon_{\text {narrow }}>=(3.76 \pm 0.23) \times 10^{-3} & \chi^{2}=7.7 \\
<\varepsilon_{\text {mid_narr }}>=(4.29 \pm 0.23) \times 10^{-3} & & =14.5 \\
<\varepsilon_{\text {mid_wide }}>=(4.04 \pm 0.23) \times 10^{-3} & & =33.0 \\
<\varepsilon_{\text {wide }}>\quad=(3.19 \pm 0.23) \times 10^{-3} & & =30.0
\end{array}
$$

for 13 degrees of freedom. In this case, only the narrower bunches had a polarization profile that was consistent with being constant. Again, the average polarization differed for the different sets; the mean is $(3.82 \pm 0.11) \times 10^{-3}$ with $\chi^{2} /$ d.f. $=4.19$. Thus there is evidence from both scans in the blue beam for fill 5236 that the polarization was bunch width dependent. 

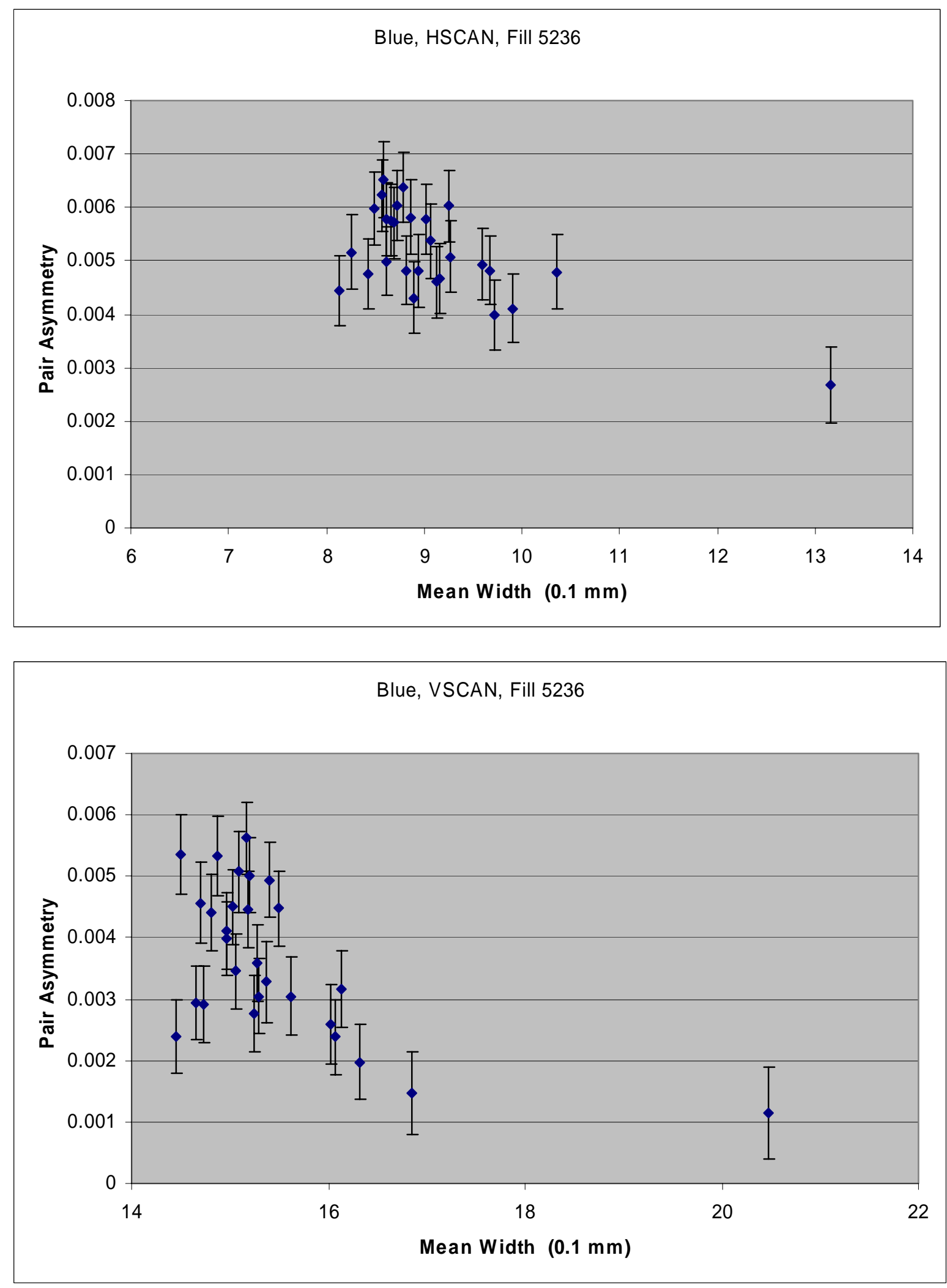

Fig. 16. Plot of the asymmetry of bunch pairs as a function of their mean width for the blue beam in fill 5236; horizontal (upper) and vertical (lower) scan results are shown. 


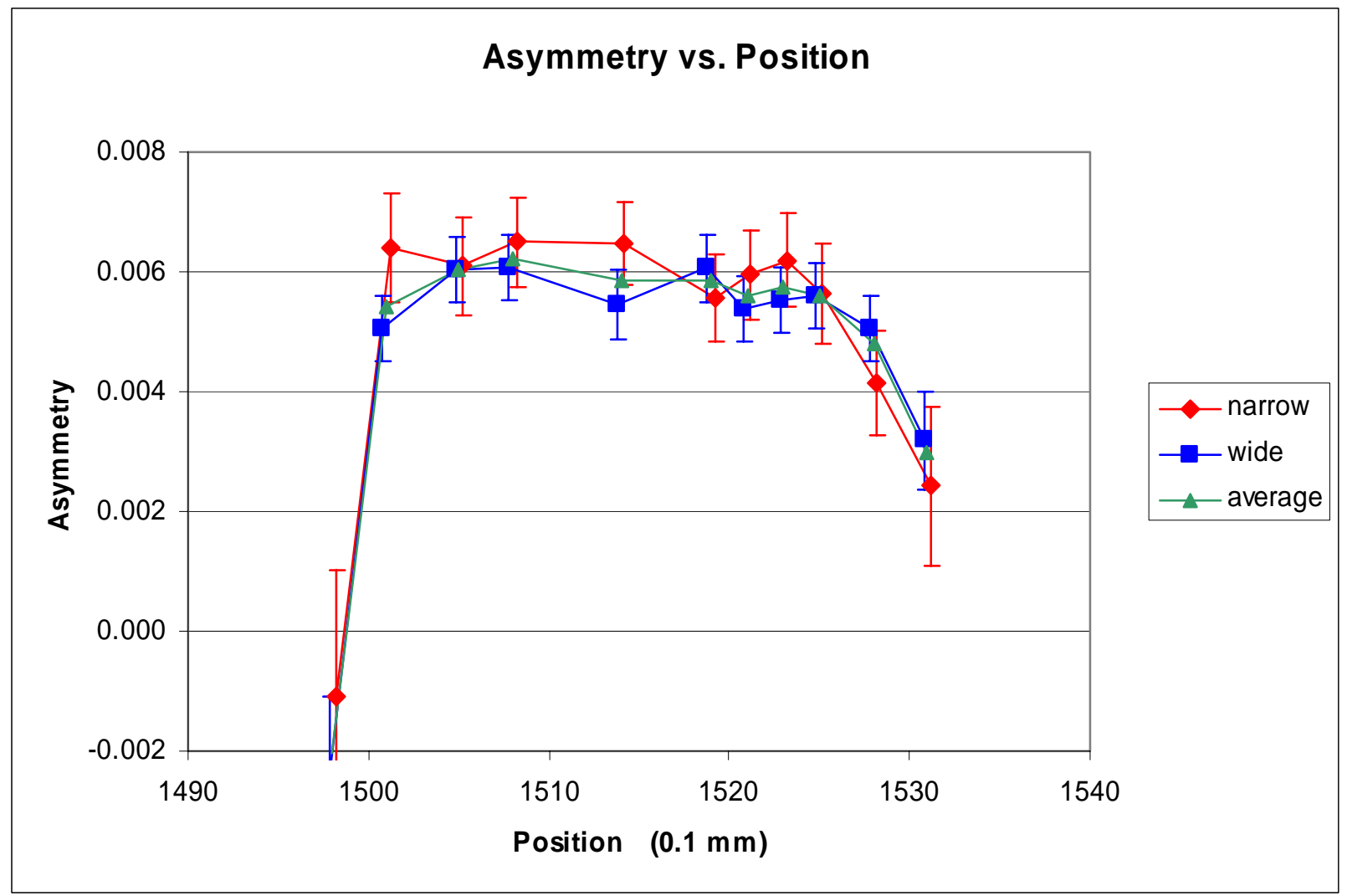

Fig. 17. Plot of CNI polarimeter left-right asymmetry for selected narrow and wide bunches for the yellow beam vertical scan in fill 5341. The narrow bunches have systematically higher asymmetry than the wider bunches.

A similar analysis was performed for the yellow vertical scan in 5341, selecting the two separate groups. A total of 17 bunches were found to have rms widths less than $0.76 \mathrm{~mm}$; this group was supplemented with three (relatively narrow) bunches to make a total of $10+$ and $10-$ bunches for one set $(\operatorname{pos}=17,25,27,43,51,65,73,75,91,105 ; \operatorname{neg}=7,15,29,37,55,63,71,77,85,103)$. The remaining bunches were placed in the other set ( $\operatorname{pos}=1,3,9,11,33,35,41,49,57,59,67,81,83,89$, $97,99,107 ; \mathrm{neg}=5,13,23,31,39,45,47,53,61,69,79,87,93,95,101,109,111)$, and bunches 21 and 19 were omitted. Asymmetries were computed with the square root formula, and the results are shown in Fig. 17. In this case, the wider bunches have a polarization profile which is consistent with the narrower bunches, though the narrower ones may have a slightly larger average asymmetry. 


\section{Summary}

On the basis of the five CNI polarimeter target scans from run-4, the following conclusions are drawn:

- One bunch (\# 21) had a significantly different intensity profile from the others in all five scans, presumably caused by its being perturbed in order to measure the beam tune during acceleration.

- The bunch centroids seem to be very nearly constant for each beam and fill studied.

- The distribution of bunch widths differed from fill to fill and beam to beam. In some cases, there were a few wider than normal bunches (Figs. 2 and 3). There were wide (Fig. 2) and narrow (Fig. 3) distributions. In one case, there was a two-peaked width distribution (Figs. 3 and 6).

- There were unusual bunch shapes in a few cases (Fig. 5).

- For one beam and fill, there was a strong correlation of the horizontal and vertical bunch widths (Fig. 7), but the widths were not proportional.

- There is reasonable evidence that the polarization has a bunch-width dependence for some fills, with the widest bunches having the lowest measured asymmetries.

and from the WCM measurements:

- The bunch longitudinal centroids seem to be spaced at constant time difference within measurement uncertainties.

- The longitudinal bunch shapes differed, some with maxima offset from the bunch center, while others were wide, and one (\# 105) very wide.

- There appears to be some instrumental effect that causes a width-dependence to the measured areas in either the CNI polarimeter or the WCM (or both). Reducing all CNI measurement times or assuming a loss of CNI counts proportional to rates did not lead to proportionality of the areas for all bunches. 


\section{Acknowledgements}

I wish to thank my many colleagues in C-AD at Brookhaven National Laboratory, the RHIC Spin Collaboration, and STAR for their suggestions, questions, and help with understanding the data. Special thanks to Osamu Jinnouchi and Angelika Drees for providing me with the raw polarimeter and Wall current monitor data, to Thomas Roser for suggesting the comparison of areas in Fig. 10, and to David Underwood and Robert Cadman for many helpful discussions.

\section{$\underline{\text { References }}$}

1) O. Jinoucchi, private communication.

2) A. Drees, private communication. 